\title{
Recognition of sketching from surface electromyography
}

DOI:

10.1007/s00521-017-2857-3

\section{Document Version}

Accepted author manuscript

Link to publication record in Manchester Research Explorer

\section{Citation for published version (APA):}

Chen, Y., Yang, Z., Gong, R., \& Wang, J. (2017). Recognition of sketching from surface electromyography. Neural Computing and Applications, 1-13. https://doi.org/10.1007/s00521-017-2857-3

\section{Published in:}

Neural Computing and Applications

\section{Citing this paper}

Please note that where the full-text provided on Manchester Research Explorer is the Author Accepted Manuscript or Proof version this may differ from the final Published version. If citing, it is advised that you check and use the publisher's definitive version.

\section{General rights}

Copyright and moral rights for the publications made accessible in the Research Explorer are retained by the authors and/or other copyright owners and it is a condition of accessing publications that users recognise and abide by the legal requirements associated with these rights.

\section{Takedown policy}

If you believe that this document breaches copyright please refer to the University of Manchester's Takedown Procedures [http://man.ac.uk/04Y6Bo] or contact uml.scholarlycommunications@manchester.ac.uk providing relevant details, so we can investigate your claim.

\section{OPEN ACCESS}




\title{
Recognition of sketching from surface electromyography
}

\author{
Yumiao Chen · Zhongliang Yang · Hugh \\ Gong · Jianping Wang
}

Received: date / Accepted: date

\begin{abstract}
The main objective of this study is to recognize sketching precisely and effectively in human computer interaction. A surface electromyography (sEMG) based sketching recognition method is proposed. We conducted an experiment in which we recorded the sEMG signals from the forearm muscles of two participants who were instructed to sketch seven basic one-stroke shapes. Subsequently, seven features of the sEMG time domain were extracted. After reducing data dimensionality using principal component analysis, these features were used as input vectors to a sketching recognition model based on Support Vector Machines (SVM). The performance of this model was compared to two other recognition models based on Multilayer perceptron (MLP) neural networks and Self Organization Feature Map (SOFM) neural networks. The average recognition rates for the seven basic one-stroke shapes of two participants achieved by the SVM-based and MLP-based models were both $98.5 \%$ in the test set, which were slightly superior to the performance of the SOFM classifier. Our results demonstrate the feasibility of converting forearm sEMG signals into sketching patterns.
\end{abstract}

Keywords Sketching recognition · Surface electromyography · Support vector machine $\cdot$ Muscle computer interface

Yumiao Chen
Fashion Institute, Donghua University, Shanghai 200051, China

$(\bowtie)$ Zhongliang Yang

College of Mechanical Engineering, Donghua University, Shanghai 201620, China

E-mail: yzl@dhu.edu.cn

Hugh Gong

School of Materials, The University of Manchester, Manchester M13 9PL, UK

Jianping Wang

Fashion Institute, Donghua University, Shanghai 200051, China 


\section{Introduction}

It is well-known that sketching is a key mode of informal, perceptual interaction that has been shown to be especially valuable for creative design tasks $[1$, $2,3,4]$. For designers, the ability to rapidly sketch objects with uncertain types, sizes, shapes, and positions is important to the creative process [1]. Thus, the analysis and estimation of sketching in this area is very significant. More natural sketching recognition is helpful for the development of electronic sketching systems [1], computer aided sketching systems [5] and sketch-based interfaces for modeling in Computer-Aided Design (CAD) systems [6]. Despite substantial progress in this area, recognizing sketching remains a difficult problem due to the fact that sketches are informal, ambiguous and implicit [7].

Several methods have been proposed for the automatic recognition of sketching. The traditional methods are based on pen and touch medium tablets, interactive pen displays, touch screens, tablet computers, and mouse to record and transmit sketching messages to computers. In comparison to the traditional input methods, the surface electromyography (sEMG)-based method for shape recognition is promising because it can provide an interaction methodology that directly senses and decodes human muscular activity as sketching input rather than requiring direct manipulation of a physical implement[8]. For example, designers attempting to sketch out some ideas anywhere and anytime may find it advantageous to be able to do so without holding a digital pen or sketching on a limited screen area. Also, a person with wrist-hand functional disorder can get back the sketching ability.

A recent trend is toward more accessible and natural interfaces. The computer vision-based method is a popular method to help recognize sketches in the air [9]. However, computer vision-based method is vulnerable to factors such as camera angle, background and lighting, which has negative effects on the recognition performance $[10,11]$. This disadvantage of the computer-vision based method is avoided by the sEMG-based method and it can provide another method for implementing sketching in the air.

The usage of myoelectric devices employing sEMG electrodes has a long history [12]. Previously, sEMG signals have been successfully used in several fields such as robot [13] and wheelchair [14] controlling, medicine [15], development of prosthesis [16,17], fatigue detection [18,19,20], force prediction [21], etc. Researchers also have used EMG to develop a new kind of human computer interface, known as Muscle Computer Interface (muCI) [22,12,23] for recognition of hand gestures [24,25], body languages [26] and facial expressions [11].

Especially, sEMG-based approaches have been successfully used for the recognition of handwriting recently $[22,27,28,29]$. In 2003, Lansari et al. [27] proposed a novel EMG-based classification approach for arabic handwriting. In their study, neural networks using a potentially damped least mean squared algorithm is used at the classification stage. In 2009, Linderman et al. [22] implemented two fundamental approaches for decoding handwriting from the EMGs. In the first approach, they reconstructed pen traces using the Wiener 
filter. In the second approach, they recognized handwritten characters from the EMG patterns and displayed them as textual fonts. In 2010, Huang et al. [28] proposed an EMG-based handwriting recognition method with dynamic time warping algorithm, which was improved by Li et al. [29] in 2013. In 2015, Okorokova et al. [30] implemented the Kalman filter to construct a dynamic model for improving reconstruction of handwriting from multichannel EMG signals, whose results show a significant improvement over the figures, previously reported by Linderman et al. [22]. The filter makes the approach suitable for real-time operations.

However, the study on recognition of sketching from electromyography is still in its infancy, although Chowdhury et al. [12] have proposed that the technology of muCI can be used for the development of interactive digital drawing tools. Perceived technical limitations [22], the paucity of models [31], and intra-class variations and inter-class ambiguities of sketches [32] may be the main reasons.

To date, no laboratory or field studies have been published to extract normal sketching patterns directly from EMG signals of hand and arm. In this study, an sEMG-based method for the recognition of seven basic one-stroke shapes from sketching was carried out. An experiment protocol was established to record the sEMG signals from the four forearm muscles of two participants who were instructed to trace and cover each printed one-stroke shape on a sketching template randomly. Subsequently, we extracted 280 time domain indices of the sEMG signals with an analysis window. We used the principal components after reducing dimensions with Principal Component Analysis (PCA) as input vectors to an Support Vector Machine (SVM) classifier. In addition, we compared the performance of the SVM-based model to those of Multilayer Perceptron (MLP) classifier and Self Organization Feature Map (SOFM) classifier.

\section{Materials and methods}

\subsection{Participants}

Two healthy male volunteers were selected to participate in the experiment. During the screening visit, all volunteers had a medical examination to eliminate any upper limb musculoskeletal and nervous diseases, and they were right-handed. Then, volunteers were required to sign a consent form with a detailed description of the experiment and complete a background questionnaire about personal information such as height and weight. De Luca [33] pointed out that the amount of subcutaneous fatty tissue among different subjects can potentially affect force-EMG signal relationship. Thus, two eligible participants with similar height and weight were recruited (see Table 1). Before the experiment, they promised not to do any forearm or hand strenuous exercise, and they were briefed on the nature, purpose, methods and risk of the study. 
Table 1 Personal information of two participants

\begin{tabular}{lll}
\hline & Subject 1 & Subject 2 \\
\hline Age & 26 & 27 \\
Height $(\mathrm{cm})$ & 172 & 175 \\
Weight $(\mathrm{kg})$ & 68 & 70 \\
\hline
\end{tabular}

\subsection{Tested shapes}

There is no definitive set of basic sketching shapes. The most common in diagrams are: line, curve, arc, rectangle, square, diamond, circle, ellipse, arrow head and triangle [34]. The most common in CAD modeling tools such as Rhinoceros (Robert McNeel Ltd., USA) are: line, curve, circle, ellipse, arc, rectangle and polygon. Multi-stroke shapes can be sketched with an arbitrary number of basic one-stroke shapes. For example, rectangle is comprised of horizontal and vertical lines. In conclusion, horizontal line, vertical line, curve, circle, horizontal ellipse, vertical ellipse and arc are seven frequently used basic one-stroke shapes, which will be selected as the tested shapes in this study.

\subsection{Sketching template design}

To allow an easy, accurate and standardized measurement of the sEMG signals of each sketching movement, a sketching template was designed. We printed these seven one-stroke shapes into a piece of A4 paper with shallow color as sketching template. The order of the shapes printed on the list of sketching template paper was randomized. Subjects were required to sketch on the paper to trace and cover each printed shape in the order of each sheet. Fig. 1 shows one example of the sketching templates.

\subsection{Apparatus}

A 12-channel digital EMG system (ZJE-II, ZJE Studio Ltd., China) was used for collecting, amplifying and transmitting the sEMG signals. It has an amplifier gain of 1000, conditioned with a digital band-pass filter between $10 \mathrm{~Hz}-500$ $\mathrm{Hz}$ with a notch filter implemented to remove the $50 \mathrm{~Hz}$ line interference. The sEMG signals underwent a 14 bit analog to digital conversion at a sampling frequency of $1000 \mathrm{~Hz}$.

The single disposable $\mathrm{Ag} / \mathrm{AgCl}$ strip electrodes $(5 \mathrm{~cm}$ in length and $3.5 \mathrm{~cm}$ in width), filled with conductive electrode paste (Jun Kang Medical Supplies Ltd., China) were used to measure sEMG activities. The electrodes can be snapped onto the EMG cable that connects it to the EMG amplifier. Before attaching the electrode, the skin in the areas under the electrodes was shaved and cleaned with alcohol and electrolyte gel to reduce skin impedance and improve the electrical and mechanical contact of the electrodes. 


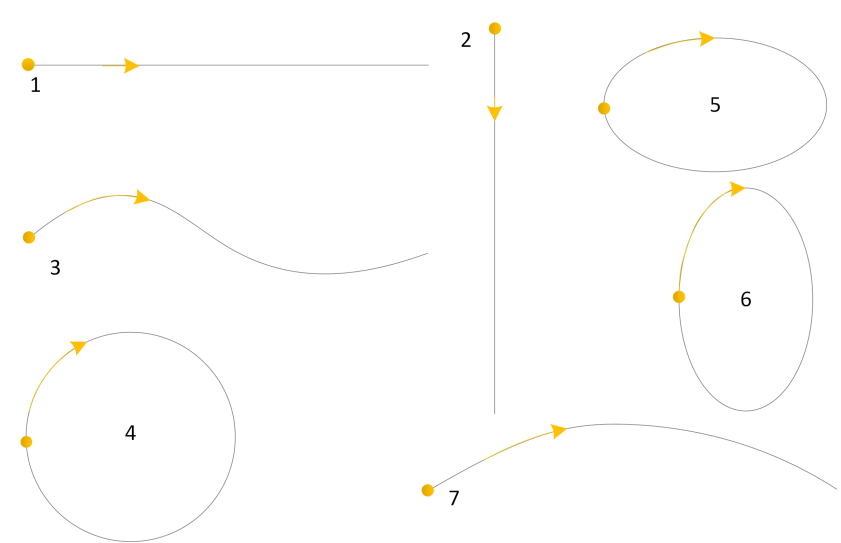

Fig. 1 One example of the sketching templates (Dots represent starting points, arrows represent directions; 1 , horizontal line; 2, vertical line; 3, curve; 4, circle; 5 , horizontal ellipse; 6 , vertical ellipse; 7 , arc)

\subsection{Selected muscles for research}

Previous researchers $[22,28]$ always recorded surface EMGs from hand muscles for handwriting recognition. However, according to our experience, sketching movements always involve forearm muscles. Thus, four forearm muscles: flexor carpi radialis (FCR), extensor digitorum (ED), extensor carpi ulnaris (ECU), extensor carpi radialis brevis (ECRB) were selected in this study. The locations of the surface EMG electrodes are shown in Fig. 2.

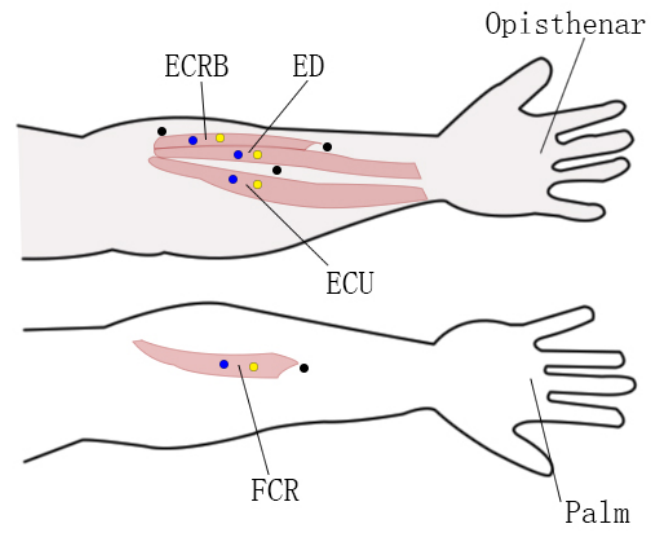

Fig. 2 Electrode placement over the right forearm muscles. ECRB, extensor carpi radialis brevis; ED, extensor digitorum; ECU, extensor carpi ulnaris; FCR, flexor carpi radialis. Here, yellow represents negative electrode, blue represents positive electrode, black represents ground electrode. 


\subsection{Experimental protocol}

The experiments were carried out for 10 days in August, 2015. They were performed from 9:00 to 12:00. We prepared 100 sheets of sketching template paper for each participant. The order of the shapes on the list of sketching template paper was randomized, with a different random order for each sheet of template paper. Each shape was sketched 100 times accumulatively. Therefore, each subject sketched 700 shapes during the whole experiment. By a trial we define a recording epoch during which a subject sketches a single one-stroke shape. The trials were paced by the timer software of mobile phone which played a beep sound in the beginning of each trial. The duration of each trial was $5 \mathrm{~s}$ of which $1-2.5 \mathrm{~s}$ corresponded to each shape sketching. The sEMG signals during extra recording time after $2.5 \mathrm{~s}$ were cut out from the analysis.

At the start of the task stage, electrodes were attached to the participants' skin and connected to the sEMG system. The locations were marked on skin with a mark pen to ensure the same locations during every session. In each session, new electrodes were attached again on the pen marks. Next, a sheet of sketching template paper was presented to the participant. Each repetition of the task was initiated with the subject holding the pen with their usual grasp pattern at a desk in front of a new sketching template. The subject was instructed to remain in this position until the maximal relaxation point and simultaneous visualization of the EMG signal were registered. At this point the beginning of the task was requested through a beep sound. EMGs of 4 muscles were simultaneously recorded. Each subject was instructed to sketch on the sketching templates to trace and cover printed shapes in order. During the sketching procedure, the right arm was in the air instead of on the table. By this way, participants can sketch more fluently. Meanwhile the extra noises of sEMG signals produced by the friction between electrodes and table can be avoided. To avoid muscle fatigue, subjects would rest for 5 minutes after each sheet of sketching template paper and the collection of EMG signals was stopped, but the electrodes were not removed until the subject finished 10 sheets of sketching template paper.

The design of the experiment was a sketching shape $(7) \times$ Repetition (10) $\times$ Day (10) within-subjects design, amounting to 700 trials per participant. Participation in the experiment took approximately 500 minutes. Fig. 3. illustrates the procedure.

\subsection{Feature extraction}

Time domain features such as Mean Absolute Value (MAV), number of Zero Crossings (ZC), number of Slope Sign Changes (SSC) and Waveform Length (WL), average EMG amplitude (aEMG), variance (VAR) and Root Mean Square (RMS) were considered for pattern recognition [35,36,37,38]. These features were extracted from 4 channels of sEMG signals during the sketching of each shape. The MAV, ZC, SSC, WL, aEMG, VAR, RMS are computed as 


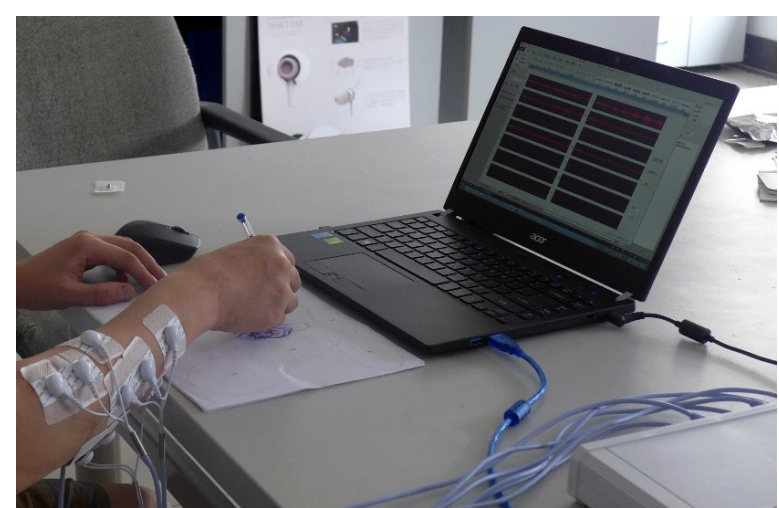

Fig. 3 A photograph of the sketching procedure

follows:

$$
M A V=\frac{1}{N} \sum_{i=1}^{N}\left|v_{i}\right|
$$

where $v_{i}$ is the voltage at the $i$ th sampling and $N$ is the number of sampling points.

$$
Z C=\sum_{i=2}^{N} \operatorname{sgn}\left(-v_{i} v_{i-1}\right)
$$

where $\operatorname{sgn}$ denotes the signum function. $Z C$ is incremented if $\left\{v_{i-1}>0\right.$ and $\left.v_{i}<0\right\}$ or $\left\{v_{i-1}<0\right.$ and $\left.v_{i}>0\right\}$ while $\left|v_{i-1}-v_{i}\right| \geqslant 0$.

$$
S S C=\sum_{i=3}^{N} \operatorname{sgn}\left[-\left(v_{i}-v_{i-1}\right)\left(v_{i-1}-v_{i-2}\right)\right]
$$

where $S S C$ is incremented if $\left\{v_{i-1}>v_{i-2}\right.$ and $\left.v_{i-1}>v_{i}\right\}$ or $\left\{v_{i-1}<v_{i-2}\right.$ and $\left.v_{i-1}<v_{i}\right\}$ while $\left|v_{i-1}-v_{i}\right| \geqslant 0$.

$$
\begin{gathered}
W L=\frac{1}{N-1} \sum_{i=2}^{N}\left|v_{i}-v_{i-1}\right| \\
a E M G=\frac{1}{N} \sum_{i=1}^{N} v_{i} \\
V A R=\frac{1}{N} \sum_{i=1}^{N}\left(v_{i}-a E M G\right)^{2} \\
R M S=\sqrt{\frac{1}{N} \sum_{i=1}^{N} v_{i}^{2}}
\end{gathered}
$$


We designed a Labview script to control the feature extraction of sEMG signals. The main channel that has the highest RMS value will be used for detecting threshold crossing, and the epoch onset of the main channel will be designated for all channels. The main channel in this paper is the channel collecting EMG signals from extensor digitorum (ED). In our study, the sliding window for detecting threshold crossing was $20 \mathrm{~ms}$ (20 samples at $1000-\mathrm{Hz}$ sampling), and the stepsize is $5 \mathrm{~ms}$. The width of the analysis window is $250 \mathrm{~ms}$ with the stepsize of $250 \mathrm{~ms}$. Rectified sEMGs from all muscles were segmented into epochs corresponding to individual tested shapes using a threshold that detected EMG bursts and designated the epoch onset. The threshold was set as 0.6 RMS during each set of tested shapes [28]. Then the epoch onset is determined when the RMS of the EMG signals crossing the threshold lasts for $300 \mathrm{~ms}$ (57 segments of sliding window for detecting threshold crossing). Then the EMG record was segmented into 2.5 s epochs (10 segments of analysis window) which represented the sketching of each shape. We can collect Segments of analysis window $(10) \times$ Time domain characteristics $(7) \times$ Tested muscles (4), amounting to 280 parameters per participant per sketching shape.

After collecting all MAV, ZC, SSC, WL, aEMG, VAR and RMS values, to reduce data dimensionality, PCA was used to preprocess the EMG data before the classification step. This would facilitates the classification, visualization, communication, and storage of high-dimensional data [39]. The number of parameters was reduced from 280 to 26 and 28 principal components for subject 1 and subject 2 respectively.

\subsection{Support Vector Machine (SVM) classifier}

Several methods are available to classify sEMG signals. We used the SVM, which is a type of machine learning method and a kernel-induced feature space function is used for the mapping of objects onto target values [40]. In the SVM, a non-linear feature mapping will allow the treatment of non-linear problems in a linear, high-dimensional space $[41,42]$. The SVM uses the idea of maximum margin classifiers for training. This decouples the capacity of the classifier from the input space and at the same time provides good generalization [43]. The approximation function used by a basic SVM is given by the following equation:

$$
f(x)=\sum_{i=1}^{l} a_{i} K\left(x, x_{i}\right)+b
$$

where $a_{i}$ represents SVM parameters to be learned from data, $x_{i}$ represents a input vector corresponding to a training object, and $K\left(x, x_{i}\right)$ represents a kernel function. The components of the vector $a$ and the constant $b$ represent the hypothesis and are optimized during training. The value of $K\left(x, x_{i}\right)$ is 
equal to the inner product of vectors $x_{i}$ and $x$ in the respective feature space. A Gaussian radial basis function is used as the kernel function in this study.

$$
K\left(x, x_{i}\right)=\exp \left[-\frac{\left|x-x_{i}\right|^{2}}{2 \sigma^{2}}\right]
$$

where $\sigma$ is a tuning parameter that controls the width of the kernel function. More detailed description of SVM can be found in [40].

\subsection{Multilayer Perceptron (MLP) classifier}

To investigate to which degree the results depended on the specific classifier used, we constructed a second classifier using the MLP neural networks. The MLP neural networks are the most commonly used feedforward neural networks due to their fast operation, ease of implementation, and smaller training set requirements $[44,45]$. The MLPs are normally trained with the backpropagation algorithm. The backpropagation rule propagates the errors through the network and allows adaptation of the hidden parameters. As one of the most common artificial neural networks (ANNs), the MLP has been widely used in pattern recognition models for sEMG signals [46]. A three-layer network consisting of one input layer, one hidden layers with a Sigmoid function, and one output layer with a Tanh function was used to set up the MLP classifier. More detailed description of the MLP can be found in [47].

\subsection{Self Organization Feature Map (SOFM) classifier}

We constructed a third classifier using the SOFM neural networks. The SOFM has been developed based on specific features of human brains cells organized in variant areas of senses presented by arranged computational maps [48, 49, 50]. The ideas of the SOFM are rooted in competitive learning networks. In competitive nets, only the weights of the winning node get updated. Kohonen proposed a slight modification of this principle with tremendous implications. Instead of updating only the winning parameter, in the SOFM nets the neighboring parameter weights are also updated with a smaller step size. This means that in the learning process (topological) neighborhood relationships are created in which the spatial locations correspond to features of the input data. In fact one can show that the data points that are similar in the input space are mapped to small neighborhoods in Kohonen SOFM layer. More details can be found in $[48,51,52]$.

Once the SOFM stabilizes, its output can be fed to a MLP to classify the neighborhoods. Note that in so doing we have accomplished two things: first, the input space dimensionality has been reduced and second, the neighborhood relation will make the learning of the MLP easier and faster because input data is structured [52]. 


\section{Results and discussion}

\subsection{Results of feature extraction}

Feature extraction and recognition algorithms had to be performed on the data from individual subjects and did not generalize to other subjects because of inter-subject variability [22]. In our experiment, sEMG signals were collected 200 times for each sketching shape $(2$ subjects $\times 100$ replications of each shape). Setting the threshold as 0.6 RMS and the Extensor digitorum (ED) as the main channel can help detect 700 trials per participant precisely. After these onsets were determined, the EMG record was segmented into 2.5 $\mathrm{s}$ epochs which represented the sketching duration of each shape. 280 parameters were successfully extracted per participant per sketching shape. Fig. 4 shows randomly selected electromyograms of the four channels for the sketching. The EMG signals of each $2.5 \mathrm{~s}$ epoch between the left and right dotted lines depicted on Fig. 4 were used in the analysis.

The PCA was carried out in SPSS software (IBM Ltd., USA). We extracted the principal components with the eigenvalues greater than 1 , which is the most common criteria in selecting principal components [51]. The number of parameters was reduced from 280 to 26 and 28 principal components for subject 1 and subject 2 respectively.

After feature dimension reduction using PCA, the dataset was randomly divided into two subsets, a training set and a test set. The $70 \%$ of the data from each day was used as the training set and the remainder of the data was used as the test set. The training set contained 490 sEMG samples, whereas the test set contained 210 samples. For our study, the linear normalization was employed as it can transform a data range to another without distortion. After linear normalization, all data was normalized into the closed interval [1, 1] [53].

\subsection{Classification results}

Three classifiers were built with the simulation software NeuroSolutions 7 (NeuroDimension Inc., Gainesville, FL, USA) on Windows 7. The accuracy rate $(A R)$ and $A R_{7}$ were used to evaluate the performance of the classifiers. $A R_{7}$ was the mean $A R$ of 7 sketching shapes for each subject.

Using SVM classifier, two termination criteria were set for the training phase: the maximum number of iterations was 2000, and the minimum mean square error (MSE) was less than 0.01. If either criterion was satisfied, the algorithms would stop. For subject 1, the algorithm converged after 65 iterations with a final MSE of 0.00996. For subject 2, the algorithm converged after 29 iterations with a final MSE of 0.00952. The confusion matrix of the sketching shapes based on the SVM classifier are shown in Figs. 5a, 5d, 5g and $5 \mathrm{j}$. 

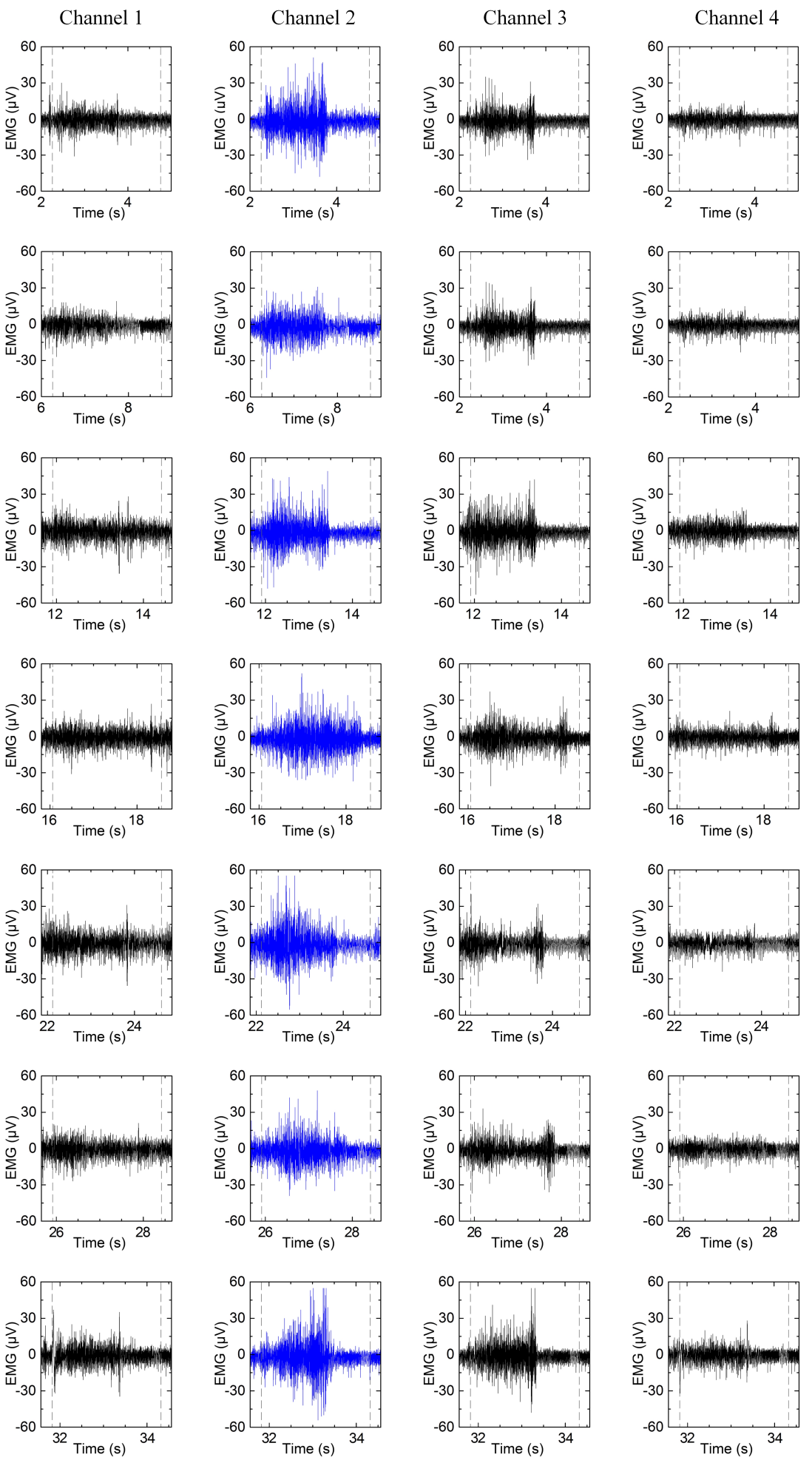

Fig. 4 Electromyograms of the four channels of the basic shapes, each panel illustrating a single sketching shape taken from a single participant (Channel 1: extensor carpi radialis brevis; Channel 2: extensor digitorum (main channel); channel 3: extensor carpi ulnaris; channel 4: flexor carpi radialis). (a) Horizontal line, (b) vertical line, (c) curve, (d) circle, (e) horizontal ellipse, (f) vertical ellipse, (g) arc. The left dotted lines represent the onsets of the $2.5 \mathrm{~s}$ epochs, and the right dotted lines represent the end points in each panel. 
As can be seen, all seven sketching shapes could be recognized with high accuracy. For the training set, the recognition Accuracy Rates ( $A R \mathrm{~s})$ are all $100 \%$. For the test set of subject 1 , the $A R$ s for horizontal line, vertical line, curve, circle, horizontal ellipse, vertical ellipse and arc are 100\%, 93.3\%, 100\%, $100 \%, 100 \%, 96.8 \%$ and $100 \%$, respectively. Furthermore, systematic misidentifications occurred: vertical line is mistaken for circle in $6.7 \%$ of cases, and vertical ellipse is mistaken for circle in $3.2 \%$. For the test set of subject 2 , the $A R$ s for horizontal line, vertical line, curve, circle, horizontal ellipse, vertical ellipse and arc are 100\%,100\%,100\%, 94.1\%, 96.8\%, 100\% and 97.4\%, respectively. Furthermore, circle is mistaken for horizontal line in $5.9 \%$ of cases, horizontal ellipse is mistaken for arc in $3.2 \%$, and arc is mistaken for curve in $2.6 \%$. Although the rate of false recognitions remains to be reduced, the SVM classifier performs very well for the training data and generalized well to the test data: for both data set, most of the sEMG patterns of the seven sketching shapes are correctly classified.

For comparison purposes, we used the same dataset and termination criteria for the MLP classifier. The number of hidden layers was set to 1 and the number of hidden layer nodes was set to 53 and 57 for subject 1 and subject 2 respectively. For subject 1, the algorithm converged after 388 iterations with a final MSE of 0.0097 . For subject 2, the algorithm converged after 224 iterations with a final MSE of 0.0098. The confusion matrix of the sketching shapes based on the MLP classifier are shown in Figs. 5b, 5e, 5h and 5k.

According to Fig. 5b, for the training set of subject 1, the $A R$ s for horizontal line, vertical line, curve, circle, horizontal ellipse, vertical ellipse and arc are $98.4 \%, 100 \%, 98.7 \%, 100 \%, 98.6 \%, 100 \%$ and $100 \%$, respectively. Furthermore, horizontal line is mistaken for circle in $1.6 \%$, curve is mistaken for arc in $1.3 \%$, and horizontal ellipse is mistaken for vertical ellipse in $1.4 \%$.

According to Fig. 5e, for the test set of subject 1 , the $A R$ s for horizontal line, vertical line, curve, circle, horizontal ellipse, vertical ellipse and arc are $97.3 \%, 100 \%, 100 \%, 100 \%, 96.8 \%, 96.8 \%$ and $97.4 \%$, respectively. Furthermore, horizontal line is mistaken for circle in $2.7 \%$, vertical ellipse is mistaken for horizontal line in $3.2 \%$, arc is mistaken for horizontal ellipse in $2.6 \%$, and horizontal ellipse is mistaken for vertical ellipse in $3.2 \%$.

According to Fig. 5h, for the training set of subject 2, the $A R$ s for the seven sketching shapes are all $100 \%$. 


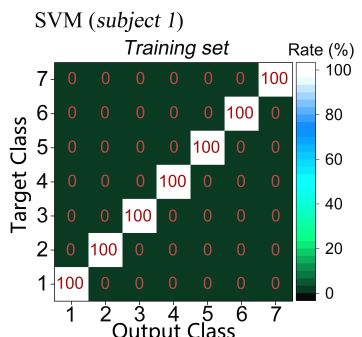

(a)

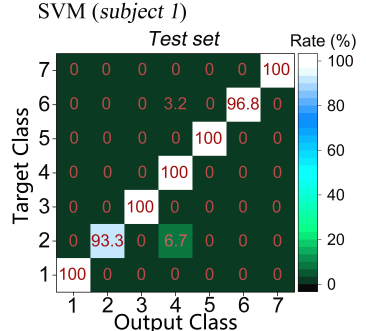

(d)

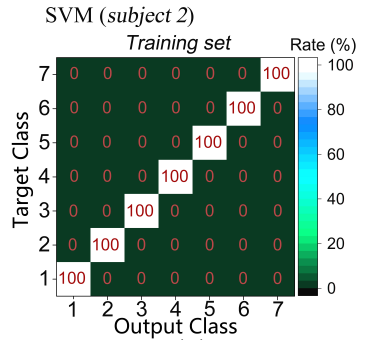

(g)

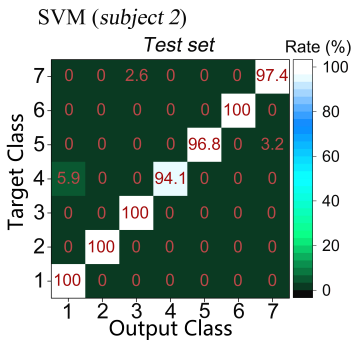

(j)

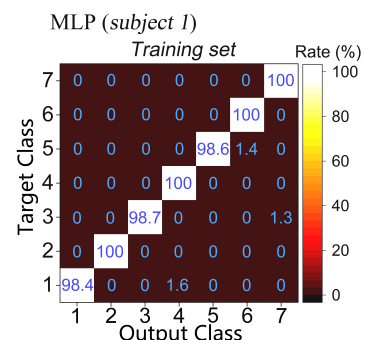

(b)

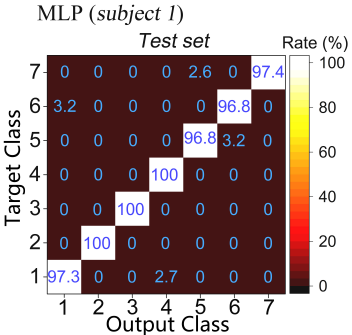

(e)

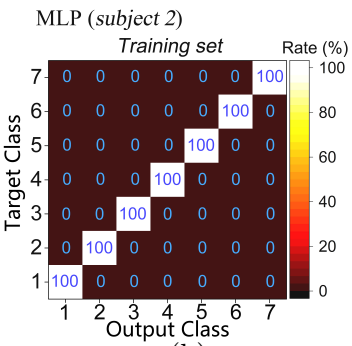

(h)

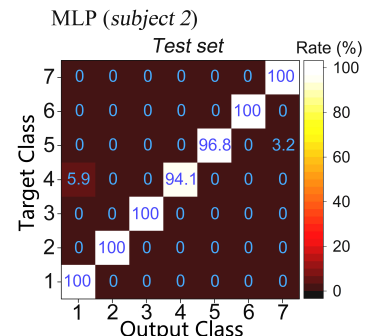

(k)

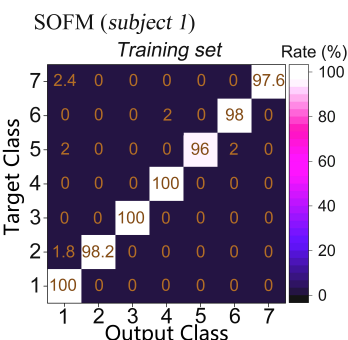

(c)

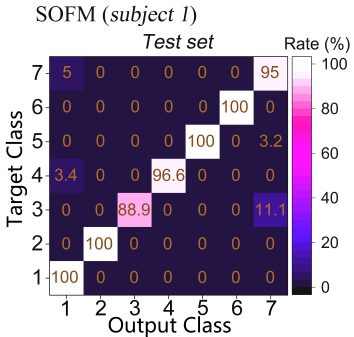

(f)

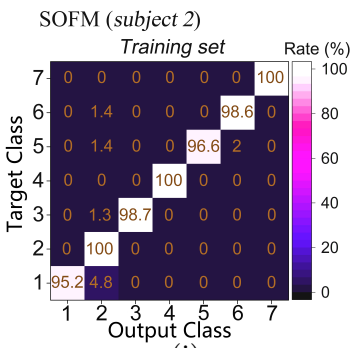

(i)

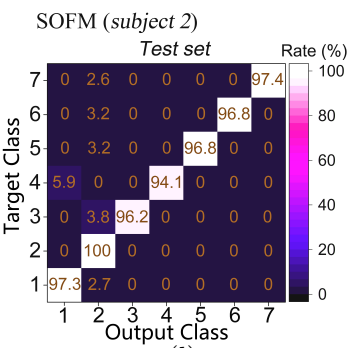

(1)

Fig. 5 Confusion matrices of seven-class sketching recognition using three classifiers (1, horizontal line; 2 , vertical line; 3 , curve; 4, circle; 5 , horizontal ellipse; 6 , vertical ellipse; 7 , arc)

According to Fig. $5 \mathrm{k}$, for the test set of subject 2, the $A R$ s for horizontal line, vertical line, curve, circle, horizontal ellipse, vertical ellipse and arc are 
$100 \%, 100 \%, 100 \%, 94.1 \%, 96.8 \%, 100 \%$ and 100\%, respectively. Furthermore, circle is mistaken for horizontal line in $5.9 \%$, and horizontal ellipse is mistaken for arc in $3.2 \%$.

We also used the same training and test set for the SOFM classifier. The properties of the SOFM classifier are shown in Table 2. The maximum epochs of unsupervised learning and supervised learning control were set as 100 and 2000 respectively. The transformation function for the hidden layer was Sigmoid function, and the transformation function for the output layer was Tanh function.

Table 2 Properties of the SOFM classifier

\begin{tabular}{llllllll}
\hline Subjects & $\begin{array}{c}\text { Number } \\
\text { of } \\
\text { inputs }\end{array}$ & $\begin{array}{c}\text { Number } \\
\text { of } \\
\text { outputs }\end{array}$ & $\begin{array}{c}\text { Number } \\
\text { of } \\
\text { hidden } \\
\text { layers }\end{array}$ & $\begin{array}{c}\text { Number } \\
\text { of } \\
\text { hidden } \\
\text { layers' } \\
\text { nodes }\end{array}$ & $\begin{array}{c}\text { Column } \\
\text { and } \\
\text { row } \\
\text { in } \\
\text { network }\end{array}$ & $\begin{array}{c}\text { Train } \\
\text { algorithm }\end{array}$ & $\begin{array}{c}\text { Neighborhood } \\
\text { shape }\end{array}$ \\
\hline 1 & 26 & 7 & 1 & 53 & $30 \times 30$ & Momentum & SquareKohonenfull \\
2 & 28 & 7 & 1 & 57 & $30 \times 30$ & Momentum & SquareKohonenfull \\
\hline
\end{tabular}

For subject 1, the algorithm converged after 701 iterations with a final MSE of 0.0099 . For subject 2, the algorithm converged after 1322 iterations with a final MSE of 0.0099. The confusion matrix of the sketching shapes based on the MLP classifier are shown in Figs. 5c, 5f, $5 \mathrm{i}$ and $5 \mathrm{l}$.

According to Fig. 5c, for the training set of subject 1, the $A R$ s for horizontal line, vertical line, curve, circle, horizontal ellipse, vertical ellipse and arc are $100 \%, 98.2 \%, 100 \%, 100 \%, 96 \%, 98 \%$ and $97.6 \%$, respectively. Furthermore, vertical line is mistaken for horizontal line in $1.8 \%$, horizontal ellipse is mistaken for horizontal line in $2 \%$ and for vertical ellipse in $2 \%$, vertical ellipse is mistaken for circle in $2 \%$, and arc is mistaken for horizontal line in $2.4 \%$.

According to Fig. 5f, for the test set of subject 1, the ARs for horizontal line, vertical line, curve, circle, horizontal ellipse, vertical ellipse and arc are $100 \%, 100 \%, 88.9 \%, 96.6 \%, 100 \%, 100 \%$ and 95\%, respectively. Furthermore, curve is mistaken for arc in $11.1 \%$, circle is mistaken for horizontal line in $3.4 \%$, and arc is mistaken for horizontal line in $2.4 \%$.

According to Fig. 5i, for the training set of subject 2, the $A R$ s for horizontal line, vertical line, curve, circle, horizontal ellipse, vertical ellipse and arc are $95.2 \%, 100 \%, 98.7 \%, 100 \%, 96.6 \%, 98.6 \%$ and $100 \%$, respectively. Furthermore, horizontal line is mistaken for vertical line in $4.8 \%$, curve is mistaken for vertical line in $1.3 \%$, horizontal ellipse is mistaken for vertical line in $1.4 \%$ and for vertical ellipse in $2 \%$, and vertical ellipse is mistaken for vertical line in $1.4 \%$.

According to Fig. 5l, for the test set of subject 2, the ARs for horizontal line, vertical line, curve, circle, horizontal ellipse, vertical ellipse and arc are $97.3 \%, 100 \%, 96.2 \%, 94.1 \%, 96.8 \%, 96.8 \%$ and $97.4 \%$, respectively. Furthermore, horizontal line, curve, horizontal ellipse, vertical ellipse and arc are 
mistaken for vertical line in $2.7 \%, 3.8 \%, 3.2 \%, 3.2 \%$ and $2.6 \%$, respectively, and circle is mistaken for horizontal line in $5.9 \%$.

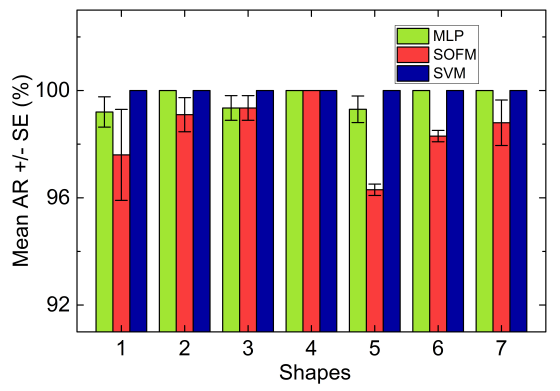

(a)

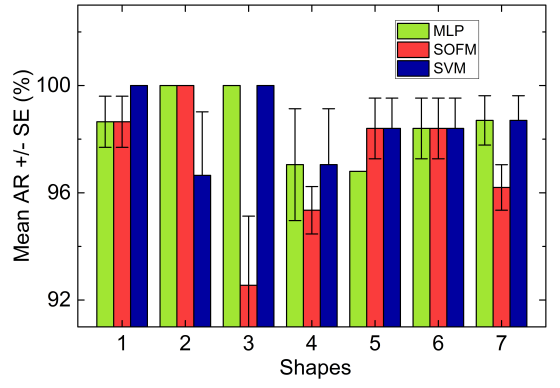

(b)

Fig. 6 Histogram bars and error bars of $A R$ for the two participants achieved by three classifiers versus type of one-stroke shapes as measured with (a) the training data and (b) the test data

The histogram bars and the error bars of $A R$ of the 7 one-stroke shapes are shown in Fig. 6. These histogram bars are the means of AR for the 2 participants. The error bars denote standard error (SE). According to Fig. 6a, the average values for the SVM classifier are all 100\%; the average values for the MLP classifier from left to right are $99.2 \%, 100 \%, 99.35 \%, 100 \%, 99.3 \%$, $100 \%$ and $100 \%$; the average values for the SOFM classifier from left to right are $97.6 \%, 99.1 \%, 99.35 \%, 100 \%, 96.3 \%, 98.3 \%$ and $98.8 \%$. According to Fig. $6 \mathrm{~b}$, the average values for the SVM classifier from left to right are $100 \%$, $96.65 \%, 100 \%, 97.05 \%, 98.4 \%, 98.4 \%$ and $98.7 \%$; the average values for the MLP classifier from left to right are 98.65\%,100\%, 100\%, 97.05\%, 96.8\%, $98.4 \%$ and $98.7 \%$; the average values for the SOFM classifier from left to right are $98.65 \%, 100 \%, 92.55 \%, 95.35 \%, 98.4 \%, 98.4 \%$ and $96.2 \%$.

The averages and standard deviation (st. dev.) of $A R_{7}$ achieved by three classifiers across subjects are shown in Table 3 . These averages are the means of $A R_{7}$ for the two participants. According to Table 3, we can conclude that the SVM classifier performs slightly better than the MLP classifier, and the SOFM classifier perform worst.

\subsection{Discussion}

The results of this paper have shown that sEMG signals can be used for the recognition of sketching. In recent years, several researchers have indicated that sEMG-based approaches are well suited for the recognition of subtle movements [22,54]. However, few studies focus on the recognition of sketching like our present study. We proposed a novel sketching recognition method based on 
Table 3 Average values of $A R_{7}$ for the 2 participants

\begin{tabular}{llll}
\hline Classifiers & Data set & Average $A R_{7}$ & st. dev. \\
\hline \multirow{2}{*}{ SVM } & Training set & $100 \%$ & $0 \%$ \\
& Test set & $98.5 \%$ & $0.18 \%$ \\
\multirow{2}{*}{ MLP } & Training set & $99.7 \%$ & $0.43 \%$ \\
\multirow{2}{*}{ SOFM } & Test set & $98.5 \%$ & $0.26 \%$ \\
& Training set & $98.5 \%$ & $0.07 \%$ \\
& Test set & $97.1 \%$ & $0.19 \%$ \\
\hline
\end{tabular}

the forearm sEMG signals. The results suggest that our SVM-based method performs very well in recognizing the seven basic one-stroke shapes from the forearm sEMG signals of four channels.

Setting the extensor digitorum as the main channel and using 0.6 RMS as the threshold, all 700 sketching epochs were detected, and all 280 parameters were extracted by the analysis window for each $2.5 \mathrm{~s}$ sketching epoch. After reducing data dimensionality using $\mathrm{PCA}$, the 26 or 28 principal components of sEMG signals were used as input vectors to the SVM classifier. This novel approach of feature extraction reveals the basis of the excellent classification result of the SVM classifier.

The fact that brain, hand and eye actions are tightly connected in the sketching process $[55,56,5]$ suggests that bioelectric interfaces potentially extract normal sketching patterns directly from forearm EMG signals. Analogously, the SVM classifier was able to recognize seven one-stroke shapes with nearly perfect accuracy. The basis of this achievement is revealed by the electromyograms (Fig. 4) which show that the four tested muscles have different sEMG amplitudes when participants sketch different basic one-stroke shapes.

The performance of the SVM classifier was slightly better than that of the MLP classifier (Figs. 5 and 6, Table 3). However, the performance differences between the two classifiers were so small that they, for practical purposes, can be regarded as equivalent. Although the SOFM classifier performed worst, the average values of $A R_{7}$ for two participants were still higher than $97 \%$, which belongs to a high recognition rate. These findings suggest that the sEMGbased sketching recognition method is robust to variations of the recognition algorithm.

Although compared with the MLP classifier, the SOFM classifier adds a competitive learning process (unsupervised learning process), which can reduce the input space dimensionality and structure the input data, the performance of the SOFM classifier was still worse than that of the MLP. The reason may be that the high complexity of a classifier may have a negative effect on the recognition accuracy rate.

For the test set, the classification performances of the three classifiers for the seven basic one-stroke shapes are different (Figs. 5 and 6). For the SVM classifier, vertical line and circle have the top two false recognition rates. For the MLP classifier, circle and horizontal ellipse have the top two false recognition rates. For the SOFM classifier, curve and circle have the top two false 
recognition rates. In conclusion, circle was always recognized worst for the three classifiers. Interestingly, circle was always mistaken for horizontal line. It can be explained that the tested muscles were not sufficient for classifying these two sketching movements.

The recognition performance attained with our non-linear approach outperform that achieved by the method based on linear discriminant analysis in [22], even though the amounts of trials, shapes and muscles we used in this paper are different from theirs $[22,30]$. In their experiment, 500 trials were collected from each participant repeating each numeric character from 0 to 9 approximately 50 times. They selected four hand muscles and four forearm muscles in their studies. It can be observed that the nonlinear methods may get higher accuracy than the linear ones, with fewer muscles and input vectors.

Overall, this demonstration may contribute to an efficient and natural way to sketch freely and precisely in computer or digital devices, which can be a viable alternative to traditional methods of sketching recognition. We envision a computer peripheral that can be used as a muscle-computer interface [12], such as an sEMG armband, in which electrical activity of forearm muscles are streamed directly to a computer where mathematical algorithms transform it into sketching shapes.

Nevertheless, our findings and the general approach have several limitations:

(1) In our study, we recognized sketching shapes from the EMG patterns. Reconstruction of sketching traces using decoding algorithm is a much more accessible and natural interface than recognition. Researchers have proposed several methods for reconstruction of handwriting from multichannel electromyographic recordings [30,22]. Analogously, EMG signals seem sufficient for the reconstruction of sketching traces, which will be the research focus of our future work.

(2) The accuracy rate of $100 \%$ is really rare in real-life, challenging classification tasks. In our future work, we will repeat the training and test procedure for each shape several times, each time with a different training data and test data, to consider the variance of test error and enhance the robustness of the method.

(3) We used sketching templates for drawing, in which we specified the sequence of shapes, the starting point and the direction of movements. This makes the problem simplified as compared to a real-life scenario, in which people have their own habits of drawing the same shape (e.g. circle can be drawn both in clockwise and counter-clockwise directions). Our future work will consider more flexible and variable ways of sketching.

(4) Subjects were required to sketch on a template with fixed dimensions (Fig. 1). However, these basic shapes can also been shown with different degrees and scales in practical drawings. Whether our method is able to recognize these additional one-stroke shapes needs further research.

(5) In this experiment, each subject was instructed to trace printed shapes, instead of free drawing. Tracing is not the same as a free drawing of shapes, 
and may reduce variability. In future studies, we therefore also plan to explore whether our method can be used to detect shapes of free drawing.

(6) Seven time domain indices of sEMG signals were extracted as features. However, too many indices may increase the number of parameters for input vectors of classifiers, which may reduce recognition rates. To further improve the robustness and accuracy, frequency domain indices and time-frequency domain indices could be additionally extracted, and we could find out the best combination of indices among them.

(7) While we recognized 7 one-stroke shapes from the EMG patterns, which are most common in diagrams and CAD modeling tools. However, there is no definitive set of basic sketching shapes. In the future work, we should enlarge the number of tested shapes and make the tested shape set more comprehensive and practical.

\section{Conclusion}

In this paper, we investigated the relationship between sketching and neuromuscular activity measured by electromyography. We built the SVM classifier in order to recognize the basic one-stroke shapes based on the corresponding sEMG measurements. We showed that the SVM classifier slightly outperforms two other classifiers. While the results are encouraging, additional research is needed to further develop the method. Further progress in this field would become useful in muscle-computer interfaces, associated with sketching.

\section{Acknowledgements}

The authors would like to thank the participants of the experiment. They also thank the editors and anonymous referees for useful comments. This study was partly supported by the National Natural Science Foundation of China (No. 51305077), the Fundamental Research Funds for the Central Universities (No. CUSF-DH-D-2016068), the Zhejiang Provincial Key Laboratory of integration of healthy smart kitchen system (2014E10014), and the China Scholarship Council (CSC). Grant Numbers: 201506630036, 201506635030.

\section{References}

1. J. Landay, B. Myers, et al., Sketching interfaces: Toward more human interface design, Computer 34 (3) (2001) 56-64.

2. M. D. Gross, E. Y.-L. Do, Ambiguous intentions: a paper-like interface for creative design, in: Proceedings of the 9th annual ACM symposium on User interface software and technology, ACM, 1996, pp. 183-192.

3. J. $\mathrm{Pu}, \mathrm{D}$. Gur, Automated freehand sketch segmentation using radial basis functions, Computer-Aided Design 41 (12) (2009) 857-864.

4. D. A. Schön, The reflective practitioner: How professionals think in action, Vol. 5126, New York: Basic books, 1983. 
5. L. Sun, W. Xiang, C. Chai, C. Wang, Q. Huang, Creative segment: A descriptive theory applied to computer-aided sketching, Design Studies 35 (1) (2014) 54-79.

6. L. Olsen, F. F. Samavati, M. C. Sousa, J. A. Jorge, Sketch-based modeling: A survey, Computers \& Graphics 33 (1) (2009) 85-103.

7. Y. Li, Incremental sketch understanding for intention extraction in sketch-based user interfaces, Citeseer, 2003.

8. T. S. Saponas, D. S. Tan, D. Morris, R. Balakrishnan, Demonstrating the feasibility of using forearm electromyography for muscle-computer interfaces, in: Proceedings of the SIGCHI Conference on Human Factors in Computing Systems, ACM, 2008, pp. $515-524$.

9. Y. Chen, J. Liu, X. Tang, Sketching in the air: a vision-based system for 3d object design, in: Computer Vision and Pattern Recognition, 2008. CVPR 2008. IEEE Conference on, IEEE, 2008, pp. 1-6.

10. Y. Zhao, Human emotion recognition from body language of the head using soft computing techniques, Ph.D. thesis, University of Ottawa (2012).

11. Y. Chen, Z. Yang, J. Wang, Eyebrow emotional expression recognition using surface emg signals, Neurocomputing.

12. A. Chowdhury, R. Ramadas, S. Karmakar, Muscle computer interface: A review, in: ICoRD'13, Springer, 2013, pp. 411-421.

13. K. Kiguchi, Y. Hayashi, An emg-based control for an upper-limb power-assist exoskeleton robot, Systems, Man, and Cybernetics, Part B: Cybernetics, IEEE Transactions on 42 (4) (2012) 1064-1071.

14. D. Andreasen, D. Gabbert, Electromyographic switch navigation of power wheelchairs, in: Annual conference of the rehabilitation engineering and assistive technology society of North America, 2006.

15. C. Disselhorst-Klug, T. Schmitz-Rode, G. Rau, Surface electromyography and muscle force: limits in semg-force relationship and new approaches for applications, Clinical biomechanics 24 (3) (2009) 225-235.

16. E. Park, S. G. Meek, Adaptive filtering of the electromyographic signal for prosthetic control and force estimation., IEEE transactions on bio-medical engineering 42 (10) (1995) 1048-1052.

17. A. Hernandez Arieta, R. Katoh, H. Yokoi, Y. Wenwei, Development of a multi-dof electromyography prosthetic system using the adaptive joint mechanism, Applied Bionics and Biomechanics 3 (2) (2006) 101-111.

18. A. Subasi, M. K. Kiymik, Muscle fatigue detection in emg using time-frequency methods, ica and neural networks, Journal of medical systems 34 (4) (2010) 777-785.

19. V. P. Singh, D. K. Kumar, B. Polus, S. Fraser, Strategies to identify changes in semg due to muscle fatigue during cycling, Journal of medical engineering \& technology 31 (2) (2007) 144-151.

20. Y. Chen, Z. Yang, J. Wang, H. Gong, Physiological and subjective responses to breathing resistance of n95 filtering facepiece respirators in still-sitting and walking, International Journal of Industrial Ergonomics 53 (2016) 93-101.

21. Z. Yang, Y. Chen, Z. Tang, J. Wang, Surface emg based handgrip force predictions using gene expression programming, Neurocomputing.

22. M. Linderman, M. A. Lebedev, J. S. Erlichman, Recognition of handwriting from electromyography, PloS one 4 (8) (2009) e6791.

23. M. R. Ahsan, M. I. Ibrahimy, O. O. Khalifa, et al., Emg signal classification for human computer interaction: a review, European Journal of Scientific Research 33 (3) (2009) 480-501.

24. J. Kim, S. Mastnik, E. André, Emg-based hand gesture recognition for realtime biosignal interfacing, in: Proceedings of the 13th international conference on Intelligent user interfaces, ACM, 2008, pp. 30-39.

25. G. R. Naik, D. K. Kumar, M. Palaniswami, Multi run ica and surface emg based signal processing system for recognising hand gestures, in: Computer and Information Technology, 2008. CIT 2008. 8th IEEE International Conference on, IEEE, 2008, pp. 700-705.

26. Y. Chen, J. Wang, Z. Yang, An semg-based attitude recognition method of nodding and head-shaking for interactive optimization, Journal of Computational Information Systems 10 (18) (2014) 7939-7948. 
27. A. Lansari, F. Bouslama, M. Khasawneh, A. Al-Rawi, A novel electromyography (emg) based classification approach for arabic handwriting, in: Neural Networks, 2003. Proceedings of the International Joint Conference on, 2003, pp. $2193-2196$.

28. G. Huang, D. Zhang, X. Zheng, X. Zhu, An emg-based handwriting recognition through dynamic time warping, in: Engineering in Medicine and Biology Society (EMBC), 2010 Annual International Conference of the IEEE, 2010, pp. 4902-4905.

29. C. Li, Z. Ma, L. Yao, D. Zhang, Improvements on emg-based handwriting recognition with dtw algorithm, in: Engineering in Medicine and Biology Society (EMBC), 2013 35th Annual International Conference of the IEEE, IEEE, 2013, pp. 2144-2147.

30. E. Okorokova, M. Lebedev, M. Linderman, A. Ossadtchi, A dynamical model improves reconstruction of handwriting from multichannel electromyographic recordings, Frontiers in neuroscience 9 .

31. I. W. McKeague, A statistical model for signature verification, Journal of the American Statistical Association 100 (469) (2005) 231-241.

32. Y. Li, T. M. Hospedales, Y.-Z. Song, S. Gong, Free-hand sketch recognition by multikernel feature learning, Computer Vision and Image Understanding.

33. C. J. De Luca, The use of surface electromyography in biomechanics, Journal of applied biomechanics 13 (1997) 135-163.

34. P. Schmieder, B. Plimmer, R. Blagojevic, Automatic evaluation of sketch recognizers, in: Proceedings of the 6th Eurographics Symposium on Sketch-Based Interfaces and Modeling, ACM, 2009, pp. 85-92.

35. H. Ren, G. Xu, S. Kee, Subject-independent natural action recognition, in: Automatic Face and Gesture Recognition, 2004. Proceedings. Sixth IEEE International Conference on, IEEE, 2004, pp. 523-528.

36. G. Huang, Modeling, analysis of surface bioelectric signal and its application in human computer interaction, Ph.D. thesis, Shanghai Jiao Tong University (2013).

37. U. Baspinar, H. S. Varol, V. Y. Senyurek, Performance comparison of artificial neural network and gaussian mixture model in classifying hand motions by using semg signals, Biocybernetics and Biomedical Engineering 33 (1) (2013) 33-45.

38. J. Potvin, Effects of muscle kinematics on surface emg amplitude and frequency during fatiguing dynamic contractions, Journal of Applied Physiology 82 (1) (1997) 144-151.

39. G. E. Hinton, R. R. Salakhutdinov, Reducing the dimensionality of data with neural networks, Science 313 (5786) (2006) 504-507.

40. C. Cortes, V. Vapnik, Support-vector networks, Machine learning 20 (3) (1995) 273-297.

41. K. Zhang, S. Sun, Web music emotion recognition based on higher effective gene expression programming, Neurocomputing 105 (2013) 100-106.

42. B. Schölkopf, A. J. Smola, Learning with kernels: support vector machines, regularization, optimization, and beyond, MIT press, 2002.

43. Q.-Y. Tang, M.-G. Feng, Dps data processing system: experimental design, statistical analysis and data mining, Science, Beijing.

44. Y. Kocyigit, A. Alkan, H. Erol, Classification of eeg recordings by using fast independent component analysis and artificial neural network, Journal of Medical Systems 32 (1) (2008) 17-20.

45. E. D. Übeyli, Combined neural network model employing wavelet coefficients for eeg signals classification, Digital Signal Processing 19 (2) (2009) 297-308.

46. G. Tsenov, A. Zeghbib, F. Palis, N. Shoylev, V. Mladenov, Neural networks for online classification of hand and finger movements using surface emg signals, in: Neural Network Applications in Electrical Engineering, 2006. NEUREL 2006. 8th Seminar on, IEEE, 2006, pp. 167-171.

47. M. Gardner, S. Dorling, Artificial neural networks (the multilayer perceptron)a review of applications in the atmospheric sciences, Atmospheric environment 32 (14) (1998) $2627-2636$.

48. T. Kohonen, Self-organization and associative memory, Vol. 8, Springer, 2012.

49. L. Sadowski, M. Nikoo, Corrosion current density prediction in reinforced concrete by imperialist competitive algorithm, Neural Computing and Applications 25 (7-8) (2014) 1627-1638.

50. M. Nikoo, P. Zarfam, H. Sayahpour, Determination of compressive strength of concrete using self organization feature map (sofm), Engineering with Computers 31 (1) (2015) $113-121$. 
51. Ł. Sadowski, M. Nikoo, M. Nikoo, Principal component analysis combined with a self organization feature map to determine the pull-off adhesion between concrete layers, Construction and Building Materials 78 (2015) 386-396.

52. I. NeuroDimension, Neurosolutions user's guide.

URL http://www.neurosolutions.com/neurosolutions/help/index.html? NormalizationFile.html

53. J. Xiong, G. Zhang, J. Hu, L. Wu, Bead geometry prediction for robotic gmaw-based rapid manufacturing through a neural network and a second-order regression analysis, Journal of Intelligent Manufacturing 25 (1) (2014) 157-163.

54. X. Chen, X. Zhang, Z.-Y. Zhao, J.-H. Yang, V. Lantz, K.-Q. Wang, Hand gesture recognition research based on surface emg sensors and 2d-accelerometers, in: Wearable Computers, 2007 11th IEEE International Symposium on, IEEE, 2007, pp. 11-14.

55. V. Goel, Sketches of thought, MIt Press, 1995.

56. T. Taura, E. Yamamoto, M. Y. N. Fasiha, M. Goka, F. Mukai, Y. Nagai, H. Nakashima, Constructive simulation of creative concept generation process in design: a research method for difficult-to-observe design-thinking processes, Journal of Engineering Design 23 (4) (2012) 297-321. 